\title{
Caracterización y desarrollo de la competencia de ciudadanía en la formación de docentes de matemáticas de secundaria
}

\author{
Yuri Morales López \\ ymorales@una.cr \\ Escuela de Matemática \\ Universidad Nacional, Costa Rica
}

\author{
Marianela Alpízar Vargas \\ malpiza@una.cr \\ Escuela de Matemática \\ Universidad Nacional, Costa Rica
}

\author{
Ana Lucía Alfaro Arce \\ aalfar@una.cr \\ Escuela de Matemática \\ Universidad Nacional, Costa Rica
}

Recibido: Julio 22, 2015

Aceptado: Noviembre 19, 2015

Resumen. El objetivo de esta investigación fue determinar el conocimiento de docentes en formación, respecto a la caracterización de un buen ciudadano, al generar reflexión sobre el concepto de ciudadanía a través de actividades vinculadas con estadística y probabilidad, y cómo este concepto puede ser desarrollado en la educación secundaria. Las actividades se implementaron durante el I ciclo del 2014 en un grupo de futuros educadores de matemáticas de la carrera Bachillerato y Licenciatura en Enseñanza de la Matemática de la UNA en Costa Rica. Los profesores en formación mostraron algunas debilidades conceptuales que les restringió crear actividades más sofisticadas para enseñar los temas y promover ciudadanía en los estudiantes de secundaria.

Palabras clave: Educación matemática, formación de docentes, competencias, ciudadanía.

\begin{abstract}
The aim of this paper is determinate the knowledge of teachers in initial training regarding to the characterization of a good citizen, to generate reflection on the concept of citizenship through activities related to statistics and probability, and how this concept can be developed in education secondary. The activities were implemented during the first half of 2014 in a group of future teachers of mathematics at the career Bachelor and Licenciature of Teaching Mathematics of the Universidad Nacional in Costa Rica. Teachers in initial training showed significant conceptual weaknesses which restricted them to create more sophisticated activities to teach the issues and to promote citizenship in high school students.
\end{abstract}

KeyWords: mathematics education, teacher training, competencies, citizenship 


\subsection{Introducción}

Como parte de las peculiaridades sociales de las décadas anteriores, la educación ha tenido un desarrollo complejo y no siempre constante. Parte de la herencia histórica en las actividades de enseñanza y aprendizaje corresponde a una formación centrada en contenidos, donde fue privilegiada la separación entre materias y actividades educativas.

Junto a esto, la adopción de pruebas estandarizadas como instrumentos de valoración por excelencia, posiblemente ha contribuido para crear una brecha importante en la formación en los contenidos y la formación de ciudadanos. Esto significa que, en algunos casos, se preste mayor atención al contenido y no tanto a la formación que se realiza al comprender la matemática.

El caso de la educación matemática no está excluido de esta problemática. Es necesario que la educación formal que se ofrece en esta disciplina no se reduzca solamente a conceptos o elementos temáticos, sino que se debe preparar a la ciudadanía para que afronte problemáticas de su entorno de manera eficiente y responsable.

Así, parte de las necesidades actuales en la educación matemática consiste en determinar vías para que esta brecha sea reducida, y se pueda garantizar que la formación tenga una componente certera para la preparación del ciudadano. Para emprender esta tarea, es indispensable conocer el tipo de formación que se desea y caminos para crear actividades prácticas que involucren la competencia ciudadana.

En este documento se aborda la temática de ciudadanía como una propuesta para interpretar la ciudadanía en la educación, específicamente en la educación matemática.

Una manera de diagnosticar parte de la situación es estudiar a los docentes en formación de esta disciplina y su relación con el concepto de ciudadanía. Así, el objetivo de este artículo es determinar el conocimiento de docentes en formación, respecto a la caracterización de un buen ciudadano, al generar reflexión sobre el concepto de ciudadanía a través de actividades vinculadas con estadística y probabilidad, y cómo este concepto puede ser desarrollado en la educación secundaria.

\subsection{Marco Teórico}

\section{Concepto de ciudadanía}

Para la comprensión de este concepto, es necesario mostrar algunas interpretaciones sobre ciudadanía que han tenido diversos autores; se especifican, a manera de muestra, en el contexto costarricense.

El término ciudadanía es un concepto polisémico, pues adopta distintas definiciones y vincula distintos elementos según el contexto en que se utilice. En algunos casos, se relaciona con la política y democracia; en otros entornos se asocia a valores éticos y morales, mientras que otra forma común es asociarlo

Caracterización y desarrollo de la competencia de ciudadanía en la formación de docentes de matemáticas de secundaria. Yuri Morales, Marianela Alpízar, Ana L. Alfaro

Derechos Reservados ( 2016 Revista digital Matemática, Educación e Internet (http://tecdigital.tec.ac.cr/revistamatematica/) 
con las características de un buen individuo de una sociedad. Respecto a este concepto, Marín (2006, citado por Alfaro y Badilla, 2013 [1, pág 50]) indica que "en Costa Rica hacemos una mezcolanza terrible de corrientes y tradiciones", se usa el término indistintamente para hablar de ser respetuosos de la ley y los derechos, así como educación moral, machismo - feminismo, género y valores, por mencionar algunos.

Junto a esto, un asunto fundamental para la comprensión es que el sistema educativo costarricense aún guarda muchas características de sistemas parcializados en contenidos. En este caso, una visión poco integrada de lo que se aborda en las distintas materias ha provocado que temas como ciudadanía hayan sido atendidos tradicionalmente por la asignatura estudios sociales en primaria y, principalmente en la asignatura cívica en secundaria.

Para Montoya [5, pág 2], quien participó en la redacción de la fundamentación de los Programas de Cívica, la ciudadanía trata de "convivir en sociedad dentro de un marco democrático de Estado de Derecho y de respeto a los derechos en su sentido más pleno" y en relación con los valores asociados se pueden mencionar "la justicia y la equidad; la autonomía, entendida como antítesis del autoritarismo o el mero tutelaje; la tolerancia y el respeto y aprecio de la diversidad".

Este autor señala que la ciudadanía se puede visualizar desde la democracia, definiéndola como:

Una ciudadanía que reconoce, fundamentalmente, la igualdad de los derechos y deberes de todos y el consecuente respeto mutuo y el respeto por las reglas legítimamente establecidas. Quien ejerce la ciudadanía se concibe idealmente como una persona comprometida con la democracia como mejor forma de gobierno. Ese compromiso implica considerarla como el mejor procedimiento de gobernar y de dirimir las diferencias y conflictos, a través del engranaje legal e institucional que consolida y permite el funcionamiento de la sociedad en su conjunto [5, pág 7].

A partir de las nociones proporcionadas en relación con el tema de ciudadanía, en el siguiente apartado se muestra una serie de características que puede poseer el ciudadano costarricense. Se trata de una construcción propia.

\section{Caracterización de ciudadano}

Basado en lo anterior, se enuncian algunas de las características que, a consideración de los autores, debe tener un buen ciudadano; se clasifican en cuatro categorías: convivencia, criticidad, democracia y conciencia social. 


\begin{tabular}{|c|l|}
\hline Categoría & Indicador \\
\hline Convivencia & $\begin{array}{l}\text { 1. Capacidad para el trabajo en equipo. } \\
\text { 2. Ser respetuoso y tolerante con las distintas maneras de pensar, credos, religiones, } \\
\text { 3. Respetar la vida en todas sus representaciones. } \\
\text { 4. Capacidad para la resolución de conflictos. }\end{array}$ \\
\hline \multirow{2}{*}{ Criticidad } & $\begin{array}{l}\text { 5. Capacidad para opinar sobre un tema o situación que aqueja a la sociedad. } \\
\text { 6. Capacidad para buscar soluciones viables a un problema real. } \\
\text { 7. Capacidad para analizar los datos y resultados que obtiene con una óptica real y } \\
\text { objetiva. }\end{array}$ \\
\hline Democracia & $\begin{array}{l}\text { 8. Reconocer y valorar el sistema democrático como necesidad para la vida en so- } \\
\text { ciedad. } \\
\text { 10. Conocer y respetar la legislación nacional (leyes, deberes y derechos). }\end{array}$ \\
\hline conciencia & $\begin{array}{l}\text { 11. Tener conciencia de la realidad del país en ámbitos como: político, económico, } \\
\text { social, entre otros. } \\
\text { problemáticas sociales y desarrollando un sentido de pertenencia. } \\
\text { 13. Conocedor de la historia del país y de temáticas afines a su profesión. } \\
\text { 14. Mostrar solidaridad con quien la necesite. } \\
\text { 15. Ser responsable al tomar sus decisiones y a la hora de actuar. }\end{array}$ \\
\hline
\end{tabular}

Tabla 1.1: Características de un buen ciudadano costarricense (Nota: La construcción se realizó del análisis de Ley Fundamental de Educación, No. 2160. (1957), Programas de estudio de educación para la vida cotidiana. Tercer ciclo de Educación General Básica. Ministerio de Educación Pública. (2012). Programas de estudio en Matemáticas para la Educación general Básica y el Ciclo Diversificado. San José, Costa Rica, y Montoya, L. (2010). Ética, Estética y Ciudadanía - Implementación y Abordaje de los Programas de Estudio de Educación Cívica, Artes Plásticas y Educación Musical. Fundamentación teórica).

\section{La ciudadanía y las matemáticas}

En Costa Rica se han venido dando en los últimos años cambios sociales en respuesta a un mundo globalizado y, por ende, el ámbito educativo ha tenido que revisar su orientación y hacer ajustes. El Ministerio de Educación Pública (MEP) ha cambiado algunos de los programas de estudio para distintas materias; entre ellos: español para primaria, educación cívica para secundaria, matemáticas para primaria y secundaria, entre otros.

En diversos eventos se ha discutido sobre la necesidad de reorientar los procesos de aprendizaje de la matemática de tal manera que se presente a los estudiantes una cara más humana de esta asignatura, mediante la inclusión de factores afectivos, éticos, actitudinales y socioculturales (Castillo, 2010 [2]).

El 21 de mayo de 2012 se aprobaron los nuevos Programas de Estudio para Matemáticas del MEP, desde primaria hasta secundaria; con estos se busca un cambio con miras a mejorar la formación básica de la población costarricense, con una matemática que prepare para la vida (Ministerio de Educación Pública (MEP), 2012 [4]). De manera específica para las matemáticas y su papel en la sociedad, según el MEP [4, pág 18] con el programa de estudios vigente: 
Se pretende afirmar una vocación de la competencia matemática especialmente asociada a la construcción de capacidades ciudadanas esenciales para el progreso de la nación. No se trata de formar las mentes para poder realizar exclusivamente propósitos limitados como el dominio de técnicas sofisticadas de demostración o la edificación de estructuras tremendamente abstractas alejadas del entorno, o para el disfrute etéreo y privado del conocimiento. Se busca por medio de las Matemáticas apoyar la comprensión e intervención ciudadana sobre diversos contextos físicos, sociales, profesionales, científicos, culturales, y por lo tanto brindarle a los individuos condiciones para poder contribuir al progreso de la patria, dentro de un espíritu de responsabilidad y respeto.

Lo anterior deja en evidencia el énfasis que el MEP quiere dar a la formación de estudiantes de primaria y secundaria, donde hacer cálculos engorrosos y procedimientos complicados que nunca se empatan con la realidad del estudiante no sea el eje principal de la educación; sino que se utilicen como herramientas que permitan la solución y el análisis de situaciones reales que suceden a menudo en el quehacer cotidiano y, por ende, se contribuya con la formación de conductas o capacidades relacionadas con la ciudadanía.

Otra de las acciones tendientes a formar buenos ciudadanos es la inclusión de los temas transversales en las distintas asignaturas.

Para el MEP (2012, [4, pág 19]):

El propósito de incorporar estos ejes transversales se ve favorecido fuertemente por este enfoque curricular que apuntala la relación de la enseñanza aprendizaje de las Matemáticas con los entornos sociales y culturales, los que se incorporan de una forma natural en los planes de estudio.

\section{Concepto de ciudadanía en la educación matemática}

Autores como Vanegas [6] usan una caracterización para la comprensión de un desarrollo de ciudadanía en educación, directamente en matemáticas. Según esta autora, esto incluye:

- una educación en valores transversales y transdisciplinares;

- toma de conciencia política y de responsabilidad en la construcción de identidad y cohesión en los objetivos curriculares;

- la construcción de ciudadanía desde una ética comunicativa democrática;

- la construcción de ciudadanía desde el diálogo transformador y liberador;

- lleva a una ciudadanía democrática mediante prácticas matemáticas participativas solidarias;

- aprender a formar en ciudadanía a través de las matemáticas mediante prácticas matemáticas que buscan una construcción humanista de la matemática y su enseñanza.

Font, Giménez, Zorrilla y Larios [3, pág 61] indican que, por ejemplo, comprendiéndola como una competencia en la formación de educadores, la ciudadanía en el docente de matemática significa "manifestar una conducta permanente de respeto por la dignidad de la persona humana, como muestra de desarrollo de una identidad profesional sustentada en un conjunto de valores vinculados a un compromiso ético en su práctica" .

Finalmente, se ofrece al lector una construcción propia del concepto de competencia ciudadana en la educación matemática. 
Se comprenderá, de forma inicial, la ciudadanía en la educación como una competencia general, y se traduce como: La capacidad de convivir con los semejantes de manera armoniosa, respetar la democracia con sentido crítico y ser consciente del papel de los individuos en el desarrollo de una sociedad con conciencia social y ambiental, con valores definidos.

\subsection{Marco metodológico}

En este apartado se resume el procedimiento que se llevó a cabo para la recolección de los datos, los instrumentos utilizados y su análisis.

\section{Tipo de estudio y participantes}

El estudio se enmarca en el enfoque cualitativo, específicamente, de tipo descriptivo, ya que se buscaba describir el razonamiento de docentes en formación al realizar cada una de las actividades relacionadas con ciudadanía y análisis estadístico que se llevaron a cabo en el curso Inferencia Estadística (MAB401), ubicado en el cuarto nivel de la carrera Bachillerato y Licenciatura en la Enseñanza de la Matemática en la Universidad Nacional de Costa Rica, en el primer ciclo del 2014. El campo de trabajo de estos futuros docentes será el III Ciclo de la Educación General Básica y el Ciclo Diversificado (estudiantes de 12 a 17 años).

La matrícula total en el curso fue 13 personas, quienes constituyen los participantes de esta investigación. El objetivo a cumplir con el estudio fue determinar el conocimiento con el que cuentan estos educadores en formación respecto a la caracterización de un buen ciudadano, al generar reflexión sobre el concepto de ciudadanía, a través de actividades vinculadas con estadística y probabilidad, y cómo este concepto puede ser desarrollado en la educación matemática en la secundaria.

\section{Instrumentos y recolección de la información}

A través del ciclo, se llevaron a cabo tres actividades: diagnóstico oral, diagnóstico escrito y elaboración de proyectos. A continuación se describe cada una de ellas.

\subsubsection{Diagnóstico oral}

Se realizó un diagnóstico oral al inicio del curso (febrero de 2014) relacionado con elementos generales sobre el papel de la matemática en la formación de las personas; principalmente, la importancia de las temáticas de estadística y probabilidad en la formación de buenos ciudadanos.

De manera específica, se le consultó a los docentes en formación, sobre su conocimiento acerca de los Programas de Matemática vigentes del MEP en secundaria y para qué se enseña matemática en secundaria; además, sobre el significado de ser un buen ciudadano, y cómo contribuyen las áreas de estadística y probabilidad a formarlo. Por último, se les hizo reflexionar acerca de si es posible aprender ciudadanía a través de la matemática y de qué manera.

Caracterización y desarrollo de la competencia de ciudadanía en la formación de docentes de matemáticas de secundaria. Yuri Morales, Marianela Alpízar, Ana L. Alfaro

Derechos Reservados @ 2016 Revista digital Matemática, Educación e Internet (http://tecdigital.tec.ac.cr/revistamatematica/) 
El método llevado a cabo en esta actividad fue el de interrogación: la docente a cargo formulaba una pregunta y daba oportunidad para que los estudiantes aportaran sus opiniones de manera voluntaria, se trabajó con 18 preguntas generadoras.

\subsubsection{Diagnóstico escrito}

Esta actividad se realizó también durante el mes febrero de 2014 y consistió en la aplicación de un cuestionario con seis problemas relacionados con análisis estadístico. El objetivo con este instrumento fue conocer la percepción inicial, del grupo de docentes de matemáticas en formación, sobre cómo algunas situaciones-problema de estadística y probabilidades podrían ser utilizadas para formar un buen ciudadano desde su paso por secundaria.

Los problemas presentados involucraron situaciones cotidianas como: personas adultas mayores, consumo de electricidad, enfermedades como paludismo y el dengue, defunciones por enfermedades y por accidentes de tránsito y vacunación. Para cada situación-problema se generó una pregunta de contenido (estadística o probabilidad) y varias preguntas sobre el uso de estas situaciones de forma didáctica. A manera de ejemplo se adjunta una de las actividades planteadas.

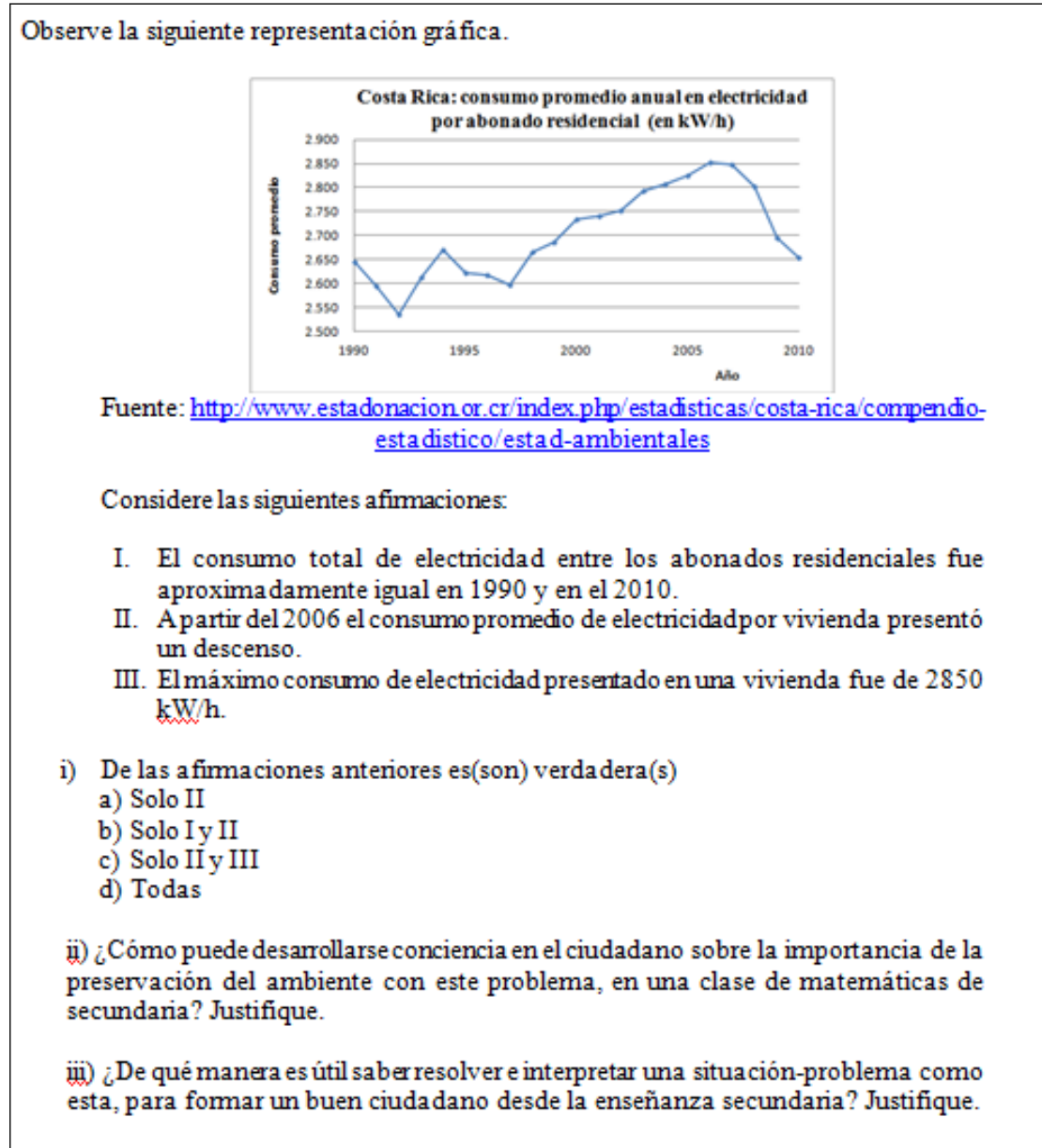

Figura 1.1: Situación problema dos. Diagnóstico escrito, curso MAB401, I ciclo (2014). 


\subsubsection{Proyectos}

Los estudiantes, en grupo de tres o cuatro integrantes, realizaron un proyecto donde debían elaborar una unidad didáctica sobre algún contenido, de las áreas de probabilidad o estadística, contemplado en el Programa de Estudio de Matemáticas del MEP vigente, abordando tanto la metodología de dicho programa así como el desarrollo de habilidades relacionadas con ciudadanía. Esta actividad se llevó a cabo al finalizar el curso MAB401, en junio 2014.

Cada proyecto debía contener, entre otros elementos:

1. Presentación y desarrollo de las actividades (situaciones problema) siguiendo las etapas de mediación pedagógica propuestas en los Programas de Estudio de Matemáticas del MEP (organización de las lecciones).

2. Respuesta a la pregunta: ¿De qué manera se desarrolla la ciudadanía en los estudiantes, mediante las actividades (situaciones-problema) planteadas?

\subsection{Análisis de datos}

Se efectúo una descripción detallada con los datos obtenidos con cada una de las actividades realizadas en el curso mencionado, con la intención de encontrar similitudes de pensamiento entre los educadores en formación acerca de la competencia ciudadanía. Así como determinar sus debilidades en cuanto al uso de actividades cotidianas para desarrollar contenidos matemáticos y relacionados con la formación ciudadana. Se expone la información del diagnóstico oral y escrito, y por último; el trabajo realizado en grupos (Proyecto). Se aclara que cuando se cita la opinión de alguno de los estudiantes en formación se le llamó alumno, sin embargo no se reporta solo la opinión de uno de ellos, sino la más representativa entre todo el grupo.

\subsubsection{Diagnóstico oral}

Cuando se le consultó a docentes en formación sobre la importancia de la matemática en la sociedad, se determinaron respuestas bastantes generales sobre la aplicación en la vida tales como en el comercio y en las finanzas, que de forma habitual son correctas. Los estudiantes señalaron claramente la importancia de la matemática como una estructura de razonamiento, útil para resolver problemas más allá del contenido matemático, propiamente. Cuando se consultó por la estadística y probabilidad las respuestas apuntaron hacia aplicaciones conocidas por ellos como juegos de azar, estadísticas de deportes, accidentes automovilísticos, entre otros. Por ejemplo:

\section{Ejemplo 1.1}

Alumno: Tal vez para medir la probabilidad de tener éxito en un proyecto y dependiendo de qué zona se maneje, qué probabilidad tengo de tener éxito.

${ }^{a}$ Se citan las intervenciones de los docentes en formación como Alumno; sin embargo, no todas las intervenciones corresponden a una misma persona. 
Respecto al concepto de buen ciudadano, los profesores en formación inicialmente señalaron varias de las obligaciones vinculadas al ejercicio de la ciudadanía. Es decir, respetar las leyes, ejercer el sufragio, no botar basura. Poco a poco se reflejaron en el discurso de los estudiantes valores generales del ciudadano: respeto, perseverancia, responsabilidad y solidaridad. Según los educadores en formación, esto se puede traducir en el aula; por ejemplo, cuando se pasa lista de asistencia (responsabilidad) o cuando se trabaja en grupo (tolerancia). Es destacable que los estudiantes indiquen la necesidad de formar grupos de apoyo a los gobiernos municipales para poder generar tareas comunes en relación con reciclaje, recolección de desechos, entre otros.

A la cuestión si la educación matemática puede contribuir a formar buenos ciudadanos, algunos restringieron sus aportes a la educación financiera (inversión, préstamos, entre otros). Otros fueron más críticos al señalar que la matemática, como contenido en sí misma, no necesariamente forma buenos ciudadanos, pues se puede aprender matemática sin generar valores, al menos conscientemente. Por ejemplo:

\section{Ejemplo 1.2}

Alumno: Yo pienso que sí puede contribuir, pero decirle que con solo enseñarle matemática la persona se vuelve buen ciudadano no es correcto. Sí, porque ayuda a crecer a nivel profesional y a cuestiones de razonamiento, da muchos beneficios,... pero no se puede decir que solo con matemáticas se desarrolla ese ciudadano.

Cuando se indagó específicamente por estadística y probabilidad en la formación de ciudadanos, se señaló el poder de estas áreas para llevar la información a las personas (de forma descriptiva) y la posibilidad que ofrece de tomar mejores decisiones (optimización de recursos, cuido del agua, manejo de desechos, aporte a la seguridad social, salud, y tránsito).

Señalan que parte de la formación en estas áreas es indispensable para poder compartir información relevante sobre la realidad y poder modificarla. Por ejemplo, hacer comprender a las personas que la falta de agua no es un asunto eventual o de pura intuición: sino que se debe educar para cuidar las nacientes y el agua disponible. Señalan, finalmente, la gran responsabilidad que reside en las instituciones de educación secundaria y por ende en los educadores.

\section{Ejemplo 1.3}

Alumno: Nosotros vamos a formar personas, no solamente matemáticamente, también tenemos que inculcar esos valores a esas personas, no solamente ir a la pizarra, aplicar un ejercicio matemático y ya. No?, también tenemos que orientar con responsabilidad la formación.

\subsubsection{Diagnóstico escrito}

Como se indicó en la metodología, este diagnóstico consistió en la ejecución de seis actividades comunes. Para efectos de esta investigación, se realiza el análisis de una de ellas, donde se logró determinar mayor incidencia en el concepto de ciudadanía. La actividad seleccionada fue la número 2, la cual se describe en la figura 1 (presentada anteriormente 1.1). 
Respecto a la pregunta vinculada con el contenido propiamente estadístico, se evidenció una importante debilidad en la interpretación de gráficas, pues no pudieron realizar la deducción correcta.

Cuando se consultó sobre la veracidad de las afirmaciones, muchos de los educadores en formación señalaron que todas eran correctas, aunque es evidente que ni la primera afirmación ni la tercera son correctas. En general, esto indica que no se ha considerado apropiadamente la información y confirma que existen problemas conceptuales asociados a construcción e interpretación de gráficas, medidas como el promedio, valores máximos y mínimos.

El problema fundamental hallado es que al existir debilidades en el dominio de los contenidos vinculados a la estadística, es difícil considerar la utilidad que puedan encontrar y, el evidente problema que se construyan conceptos erróneos, aunque lo relacionado con la competencia ciudadana sea útil.

Cuando se consultó sobre cómo puede desarrollarse conciencia en el ciudadano sobre la importancia de la preservación del ambiente con este problema, en una clase de matemáticas de secundaria, las afirmaciones realizadas estuvieron vinculadas a la producción de preguntas generadoras; además, tratar de concienciar sobre los recursos que se consumen para generar electricidad.

\section{Ejemplo 1.4}

Alumno: El tema puede ser abordado llevando un video o información que ejemplifique mejor los impactos del consumo excesivo de electricidad en el ambiente comparándolo con datos estadísticos, o bien asignar a los estudiantes la investigación del tema para luego comentarlo en general y así concientizar.

Si bien muchos realizaron aportaciones sobre la importancia del consumo responsable y las acciones que deben realizarse, pocos definieron claramente actividades para la clase de matemática. Por ejemplo,

\section{Ejemplo 1.5}

Alumno: No utilizando más de un aparato electrónico a la vez, apagar las luces durante el día, utilizarlas solo por las noches, cuando sea necesario.

Cuando se juzga la respuesta anterior, se debe advertir que el futuro profesor de enseñanza de la matemática está haciendo una valoración bastante alejada de su profesión y, aun cuando las afirmaciones que realiza podrían ser útiles, estas reflejan poca capacidad de vincular la actividad profesional con el contexto. Este tipo de aporte fue el común entre el grupo de estudiantes en formación.

En otros casos, persiste un problema importante del nivel matemático y estadístico.

\section{Ejemplo 1.6}

Alumno: Con algo tan sencillo como una regla de tres, se puede averiguar qué tanto se preserva o no el ambiente en unos 15 años.

Finalmente, respecto a la utilidad de saber resolver e interpretar una situación-problema como esta, para formar un buen ciudadano desde la enseñanza secundaria, muchos señalaron que es útil para 
hacer que las personas concienticen sobre el consumo eléctrico, ahorro de energía y preservación de los recursos naturales, como por ejemplo el agua.

Solamente un estudiante valoró que este tipo de actividad puede ser utilizado como patrón para buscar soluciones a distintos problemas, no solamente el de consumo excesivo.

\section{Ejemplo 1.7}

Alumno: Si desde la enseñanza secundaria se saben resolver e interpretar situaciones-problemas como esta, ayuda a que las personas realicen comparaciones entre las situaciones planteadas y los problemas que las mismas generan, lo cual llega a ser útil en la toma de decisiones respecto a su forma de vida, y ayuda a formar un ciudadano consciente de la realidad actual y activo a participar en los aspectos que mejoren el país.

\subsubsection{Proyectos}

En total cuatro grupos presentaron sus unidades didácticas; se reporta lo referente a las actividades planteadas en la unidad y su relación con la competencia ciudadanía. Se describe a continuación el trabajo realizado por cada uno de los grupos.

\section{Grupo G1.}

Este grupo cita al Programa de Estudios del MEP en cuanto a la relación que existe entre los problemas planteados en la clase y la cotidianidad, de manera específica en el tema de estadística y probabilidad.

"En el siglo XXI se requiere de personas capaces de comprender, interpretar y usar la información para entender la realidad, resolver distintos problemas y tomar decisiones inteligentes. Los temas de la Estadística y la Probabilidad son cada día un requisito para poder comprender lo que pasa en el mundo y poder actuar" (MEP, 2012 [4, pág 55]).

Consideran que se debe formar a ciudadanos con cultura estadística y, por ende, se deben crear actividades orientadas a ese fin.

\section{Actividad propuesta por los estudiantes de G1:}

Grupo 1: "Supongamos que entre Pedro y Carlos compran una caja con 17 manzanas pagando ambos la mitad del dinero para comprarla, pero al repartirlas notan que si cada uno toma 8 de ellas, quedaría una más sin repartir, por lo que deciden lanzar una moneda al aire para evitar discusiones sobre quién se llevaría la manzana restante. ¿Qué sería más fácil obtener escudo o corona?"

La principal razón de esta actividad es tomar en cuenta que no es posible determinar la mejor opción para elegir, puesto que algunos grupos afirmarían que es escudo mientras que otros dirán que es corona, esto depende de los lanzamientos de cada grupo 
El G1 considera que esta actividad desarrolla valores importantes en la formación de ciudadanos, de manera específica, comentan: el problema planteado se relacionará con la resolución de conflictos, en donde los involucrados en la situación no confrontan el problema de manera violenta, más bien, buscan una solución efectiva, dejando al azar decidir por ellos quien se llevaría la manzana restante. Debido a los problemas constantes de violencia, las agresiones y problemas similares vividos en la sociedad, una situación igual de favorable para ambos podría ser una manera sencilla de resolver pequeñas discusiones sin necesidad de tener una opinión de una persona externa o de agravar aún más la situación.

La actividad realizada por este grupo se puede ubicar en la categoría de convivencia descrita en la tabla 1.1 del marco teórico, ya que promueve la tolerancia y la resolución de conflictos de manera pacífica. Se puede destacar que los estudiantes plantean una situación que pretende desarrollar aspectos que son citados en el Programa de Estudios del MEP como el azar.

\section{Grupo G2.}

El objetivo general de su actividad fue inculcar conciencia ambiental en los estudiantes mediante el uso de gráficas y tabulaciones para el análisis de la información.

Este grupo propone dos actividades bajo el título general ¿Qué hacemos los costarricenses con los residuos?, la primera presenta información acerca de la Separación porcentual de residuos por provincias según municipalidades durante los años 2012 y 2013 por medio de una tabla y se plantean una serie de preguntas relacionadas al análisis de los datos y a la representación gráfica que se puede realizar, por otra parte, en la segunda actividad se propone a los estudiantes (de secundaria) realizar un cuestionario a todos sus compañeros de clase acerca de si se recicla en su hogar y el tipo de material que reciclan.

Los integrantes del G2 buscan, con las actividades planteadas, según ellos mismos citan: desarrollar en los estudiantes uno de los temas trasversales propuesto por el MEP; a saber: cultura ambiental para el desarrollo sostenible.

El trabajo realizado por este grupo se ubicaría en la categoría conciencia social de la tabla 1.1, ya que trata el tema de reciclaje y la disminución de la huella que le deja el ser humano al planeta, pretenden que, con la actividad, los estudiantes de secundaria tomen conciencia de la realidad en que se vive $y$, por ende, se comporten de manera más responsable al tomar sus decisiones; además se desarrollan temas como representación tabular, representación gráfica y análisis de las mismas.

\section{Grupo G3.}

Los integrantes del grupo G3 tratan la importancia de la estadística en la vida cotidiana. El objetivo de la actividad fue resaltar el papel que tiene la estadística como herramienta para el análisis de información de diferentes temáticas.

La primera parte de la actividad es un pequeño cuestionario que deben realizar los estudiantes de secundaria a sus familiares acerca del significado de estadística y su uso en la cotidianidad; en la clase se deben discutir las respuestas dadas a dicho cuestionario. Por otra parte, utilizan estadísticas relacionadas con causas de accidentes mortales en carretera para desarrollar el tema de representación gráfica. 
Este mismo grupo plantea otra actividad relacionada con el desarrollo histórico de la estadística donde la creatividad de los estudiantes se pone a prueba en la representación de fragmentos históricos.

Los integrantes de este grupo consideran que sus actividades cumplen con los lineamientos establecidos por Vanegas y Giménez (2011 [6]) ya que, entre otras cosas, fomentan el reconocimiento de procedimientos matemáticos en contextos reales, utilizan elementos históricos e impulsan la cooperación.

Las actividades propuestas por este grupo se enmarcan en la categoría conciencia social de la tabla 1.1, ya que inducen a la toma de decisiones de manera responsable, al hablar de las causas de accidentes de tránsito; además, consideran la historia como un pilar fundamental para desarrollar el tema estadística lo cual está en sintonía con lo establecido en los programas de estudio del MEP.

\section{Grupo G4.}

El tema estadístico a desarrollar fue variables cuantitativas y distribuciones de frecuencias y uno de sus objetivos fue conjeturar algunas ideas en donde se presentan las variables cuantitativas en la vida cotidiana.

La actividad planteada por este grupo fue que los estudiantes de secundaria, en grupos de cinco personas, y con ayuda de instrumentos de medición, debían medir alguna parte de cuerpo de los compañeros, crear una tabla, una representación gráfica y luego compartir resultados. Este grupo considera que con el planeamiento de su actividad se desarrolla el derecho y la disposición de participar en una comunidad, a través de la acción autorregulada, inclusiva, pacífica y responsable, con el objetivo de optimizar el bienestar público.

Después de participar en las actividades propuestas dentro de la unidad didáctica planteada, los integrantes del G4 consideran que con ella se fortalecen valores en los cuales los jóvenes aprenden sobre el trabajo en grupo, la solidaridad, el respeto hacia quienes lo rodean y, por ende, de ciudadanía, lo cual los colocaría en la categoría conciencia social; además de desarrollar temáticas como representación gráfica y tabular.

De manera general, los estudiantes del curso MAB401 reconocen la importancia y necesidad de desarrollar habilidades relacionas con ciudadanía en las aulas de secundaria; consideran que los temas transversales orientan el desarrollo de dichas habilidades, lo cual coincide con lo planteado por el MEP en sus programas de estudio.

La categoría de mayor predilección a desarrollar en las actividades planteadas, por los profesores en formación, fue conciencia social, lo cual es esperanzador, ya que la sociedad costarricense pasa actualmente por una crisis social donde los valores se han ido perdiendo; los participantes consideran que es importante inculcar valores como el respeto y la tolerancia, los cuales deben ser parte del desarrollo de cada ciudadano y proponen promoverlos, desde las aulas de secundaria, por medio de la asignatura matemática; ya que como docentes no solo deben formar a los estudiantes en conceptos teóricos sino darles herramientas que les permitan desarrollarse en la sociedad.

A manera de síntesis, los cuatro grupos propusieron actividades-problema que pudieron resolverse con temas de estadística y probabilidad y que permitieran que los estudiantes de secundaria desarrollaran distintas habilidades que se plantean en los Programas de Estudio de Matemáticas del MEP. Este hecho se valora positivamente por los autores, dado que los encargados de realizar las actividades están 
en formación y aun no tienen mucha experiencia en la creación de unidades didácticas y problemas contextualizados. Además, enfocaron, sus actividades, en desarrollar la ciudadanía desde la educación media costarricense en aspectos de convivencia con otros seres humanos y el medio ambiente.

Se considera que las actividades de los docentes en formación (no poseen experiencia suficiente enseñando en los salones de secundaria), aunado a que la metodología de los Programas de Estudio del MEP plantea una nueva forma de enseñar y aprender matemáticas, no profundizaron en temáticas relacionadas con probabilidad y estadística, ya que casi todos se enfocaron en la elaboración de tablas y gráficas y dejaron de lado el análisis de situaciones y la toma de decisiones. En cuanto al desarrollo de la ciudadanía, algunas de ellas no fueron tan pertinentes o no quedó claramente plasmado cómo la promueven, plantearon situaciones donde el trabajo cooperativo es importante, pero dejaron de lado aspectos de crítica social y democracia.

Sin embargo, según la percepción de los autores, el curso MAB401 se ubica en el cuarto nivel (bachillerato) de la carrera Enseñanza de la Matemática, donde los docentes en formación ya deberían tener una madurez académica suficiente que les permita realizar mayor análisis, discusión y razonamiento en las actividades propuestas para enseñar las temáticas de la asignatura en secundaria, para ahondar y relacionar la educación matemática con el contexto social, ambiental, económico, etc., del país. No solo deben quedarse con el abordaje de un concepto sino que deben relacionar diversos conceptos entre sí, con otras asignaturas y con la vida cotidiana.

Se esperaba que en las actividades planteadas se desarrollaran diversos conceptos de probabilidad y estadística con un nivel de profundidad que le permita al estudiante analizar diversas situaciones y no solo colocar los datos en una tabla o gráfica. En cuanto a ciudadanía se esperaba que no solo el trabajo en equipo se viera reflejado sino que se abordaran temáticas clave de la sociedad costarricense.

\subsection{Conclusiones}

Dentro de las principales conclusiones que se obtuvieron del estudio realizado con los educadores en formación, en el curso MAB401, perteneciente al cuarto nivel de la carrera Enseñanza de la Matemática de la Universidad Nacional de Costa Rica, se destacan las siguientes.

En el diagnóstico oral, los profesores en formación, al estar juntos dentro del salón de la clase donde se desarrollaba el curso, realizaron una mayor discusión sobre los aspectos planteados en las distintas actividades sobre ciudadanía. Esa dinámica permitió que las opiniones fueran contrastadas o respaldadas por otros. Además, que se aclararan en el momento, los distintos puntos de vista externados. Con ello se logró que estos estudiantes en formación se expresaran correctamente al exponer sus ideas sobre lo que pensaban que era un buen ciudadano y sobre las temáticas de estadística y probabilidad ahí abordadas.

Los docentes en formación reconocen la utilidad de las matemáticas, y en particular, de la estadística y probabilidad, para formar buenos ciudadanos; mencionaron que su enseñanza permite el desarrollo

Caracterización y desarrollo de la competencia de ciudadanía en la formación de docentes de matemáticas de secundaria. Yuri Morales, Marianela Alpízar, Ana L. Alfaro

Derechos Reservados (C 2016 Revista digital Matemática, Educación e Internet (http://tecdigital.tec.ac.cr/revistamatematica/) 
de habilidades y destrezas de razonamiento, y el análisis de diferentes situaciones de la cotidianidad. Particularmente, se enfocaron en mencionar características de un buen ciudadano que coincidieron con la clasificación hecha por los autores en la tabla 1 del marco teórico. Además, reconocen la responsabilidad que tiene la educación matemática en secundaria para promover ciudadanía y, por lo tanto, el compromiso y obligación de los educadores para hacer conciencia social en los estudiantes.

En el diagnóstico escrito, se presentó la particularidad de que ninguno de los estudiantes en formación pudo interpretar correctamente la gráfica presentada en la actividad 2 (Figura 1.1) sobre un concepto estadístico básico como lo es el promedio de un conjunto de datos. Esto podría ser un indicativo de que las temáticas básicas de estadística y probabilidad deben ser abordadas con más detenimiento en los cursos de estadística en la universidad. No obstante, cuando se utilizó la misma actividad para generar ciudadanía en ellos, la discusión, análisis y razonamiento de la información fue bastante pertinente.

En relación con los proyectos realizados en grupos, las actividades propuestas resultaron interesantes y aceptables, considerando que fue una de las primeras experiencias para los docentes en formación en elaborar unidades didácticas basándose en la metodología plasmada en los Programas de Estudio de Matemática del MEP, aprobados en 2012 y pensando, quizás por primera vez, en cómo promover ciudadanía a través de la educación matemática. Sin embargo, la capacidad de razonamiento y análisis sobre el valor y potencial de las actividades es un tema que quedó para discusión futura de los estudiantes en formación y de los formadores de estos.

La creación de actividades-problema orientadas al desarrollo de la competencia de ciudadanía, como primera experiencia, para los docentes en formación, permitió discusión, y un primer acercamiento al verdadero sentido que debe lograr la educación matemática en la construcción de dicha competencia.

Se pudo apreciar, en los proyectos realizados, que la mayoría de actividades propuestas para promover ciudadanía en los estudiantes de secundaria se aglutinaron en la categoría, que denominamos en el marco teórico, conciencia social y algunas en convivencia. Ello posiblemente responde a tener conciencia de la realidad del país para solventar parte de las necesidades actuales de nuestra sociedad como aumento de tolerancia, solidaridad con los más necesitados y vulnerables, respeto a la diversidad en todas sus formas, amor por el ambiente, contribuir con la disminución de la violencia y bullying (matonismo escolar) en las aulas de primaria y secundaria, adquirir responsabilidad en las diversas actuaciones y toma de decisiones, entre otras; sin embargo, otras categorías como criticidad y democracia no se fueron tomadas en cuenta.

Finalmente, debemos mencionar que los profesores en formación tienen debilidades conceptuales importantes sobre probabilidad, estadística y didáctica matemática, que les restringe crear actividades más sofisticadas para enseñar los temas y para promover ciudadanía en los estudiantes de secundaria. Esto constituye una alerta a la universidad, para discutir sobre la manera en que se están formando los futuros educadores de secundaria, pues es deseable en estos estudiantes de cuarto nivel de la carrera Enseñanza de la Matemática, una mayor capacidad de análisis, que les permita tener claridad sobre el objetivo de la educación matemática en secundaria y cuenten con las herramientas necesarias para crear situaciones didácticas que apunten al desarrollo de las competencias necesarias en sus estudiantes de secundaria.

Reconocimientos. En este artículo fue elaborado en el marco del proyecto Análisis didáctico de prácticas docentes de matemáticas en la formación de futuros profesores de matemáticas de secundaria SIA0005-14 y del proyecto 
Ciudadanía y Formación de Profesores de Matemáticas, el cual se realiza con participación de la Universidad Nacional de Costa Rica (UNA), la Universidad de Barcelona (UB), Pontificia Universidad Católica del Perú (PUCP), Universidad Autónoma de Querétaro, (UAQ), Universidad Nacional de Villa María (UNVM), Universidad Distrital de Bogotá y Universidad de los Lagos de Chile; todos con la coordinación de la Universidad de Barcelona en España. Adscrito a las CÁTEDRAS DE INTEGRACIÓN CÁTEDRAS ANDRÉS BELLO - AUALCPI: "LA UNIVERSIDAD Y LOS PROCESOS DE INTEGRACIÓN" y se produce como uno de los resultados del convenio de cooperación internacional entre la Universidad Nacional (Costa Rica) y la Universitat de Barcelona (España) con vigencia actual 2014 - 2017.

\section{Bibliografía}

[1] Alfaro, A. y Badilla, M. La conceptualización de la educación cívica en Costa Rica: Aportes de profesionales vinculados con esta disciplina. Revista Electrónica Perspectivas, 6, 44-66. Descargado de http://www.revistas.una.ac.cr/index.php/perspectivas/article/download/5097/4882 2013

[2] Castillo, M. La educación matemática en el primer ciclo de la educación primaria, estado del arte. Proyecto Integración Centroamericana por medio de la Reforma Educativa? Fondos del Gobierno de China en Taiwán. Guatemala. 2010.

[3] Font, V., Giménez, J. Zorrilla, J. F., Larios, V. Competencias del profesor de matemáticas de secundaria y bachillerado. Barcelona, Publicacions de la Universitat de Barcelona. 2012

[4] Ministerio de Educación Pública. Programas de Estudio en Matemáticas para la Educación General Básica y el Ciclo Diversificado. San José, Costa Rica. 2012

[5] Montoya, L. Ética, estética y ciudadanía - implementación y abordaje de los Programas de Estudio de Educación Cívica, Artes Plásticas y Educación Musical. Fundamentación teórica (resumen). Ministerio de Educación Pública. (Documento en línea) Descargado de:http: //portal . oas.org/LinkClick. aspx?fileticket=\%20kcdytBZikQ4\%3D\&tabid=1862. 2010

[6] Vanegas, Y., y Giménez, J. Futuros profesores de matemáticas y ciudadanía. Universidad de Barcelona. España. Recuperado de http://www.cimm.ucr.ac.cr/ocs/files/conferences/1/schedConfs/1/ papers/2284/supp/2284-6132-1-SP.pdf. 2011. 